\title{
Construction of a Physical Factor Resource for Exposome Informatics Research
}

\author{
Christopher HAWTHORNE ${ }^{\mathrm{a}}$, Niamh QUIGLEY ${ }^{\mathrm{a}^{*}}$, Callum McCLEMENTS ${ }^{\mathrm{a}^{*}}$ and \\ Guillermo H. LOPEZ-CAMPOS ${ }^{\mathrm{a}, 1}$

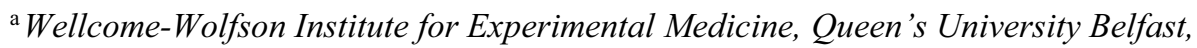 \\ Belfast, UK
}

\begin{abstract}
Exposome research is focused on all the exposures individuals experience during their lifetime and how it shapes their health and development of disease. The chemical and biological aspects of the exposome are readily available in data formats. In comparison there is a lack of data frameworks available for physical factors (e.g. noise, lighting, electromagnetic fields) and their biological relationships which would allow a greater understanding of the contribution of the physical environment on disease development and burden. We present the construction of a prototype that captures knowledge on physical factors and their interactions with genes and diseases derived from the biomedical literature to reflect the physical exposome.
\end{abstract}

Keywords. Exposome, Physical Factors, Database, Bioinformatics, Curation

\section{Introduction}

The exposome is constantly changing during an individual's lifetime in comparison to its more stable counterpart, the genome [1]. The exposures which constitute an individual's exposome can be divided into various types ranging from biological i.e. infections or the microbiome, chemical such as toxicants or medicinal drugs, or physical aspects comprising of temperature or noise. Exposome research is heavily reflected by its origins from previous environmental science research through a substantial focus in toxicological and chemical aspects. Whereas, physical factors such as noise and electromagnetic fields are shown to have an effect on the underlying biology $[2,3]$, they lack coverage in readily available data formats which hinders further exploration and analysis. We outline the development of a prototype resource derived from biomedical literature focusing on physical factors and their interactions with genes and human health in an effort to better represent the physical exposome.

\section{Methods}

The contents of the resource were selected using a series of literature queries and manual abstract annotations. We used MeSH terms to capture relevant physical exposome terms

\footnotetext{
${ }^{1}$ Corresponding Author, Guillermo Lopez Campos, Wellcome-Wolfson Institute for Experimental Medicine, 97 Lisburn Road, BT97BL, Belfast, United Kingdom; E-mail: g.lopezcampos@qub.ac.uk

* Equal contribution.
} 
and keywords as biological entities and used PubMed as a source of abstracts. The physical exposome terms considered were covered by the MeSH terms "Lighting", "Noise" and "Electromagnetic Fields". The query was built as: x[MeSH] AND 1950:2019[dp] AND ("gene"[TiAB] OR "protein"[TiAB]) AND english[LA] where 'x' represents our physical factor MeSH terms and additionally a human filter was selected. The number of abstracts retrieved for Lighting, Noise and Electromagnetic Fields were 37, 67 and 395 respectively. Abstracts were then manually curated by two independent researchers, aiming to mainly retrieve physical factors and genes. The resource interface was built through Shiny and ShinyDashboard to visualise and interact with the contents.

\section{Results}

Description of information flow for the construction of this prototype resource is presented in Figure 1(A). The manual curation was cross-validated to produce a coherent dataset. Biological effects of the physical factors were investigated using gene set enrichment analysis of the genes identified in the physical factors. The built user interface is displayed in Figure 1(B).

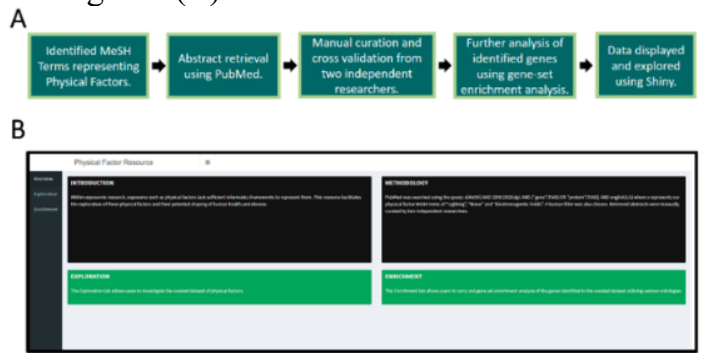

Figure 1. A) Summarisation of information flow. B) Prototype user interface of the Physical Factor Resource.

\section{Conclusions}

We have presented the construction of a prototype physical factor resource for exposome research. This resource is a first step into creating frameworks for capturing physical factor interactions with human health from the biomedical literature. This resource fills an existing gap in exposome research and allow for greater comprehension and data availability of how physical factors affect human health.

\section{References}

[1] Wild CP. Complementing the genome with an "exposome": the outstanding challenge of environmental exposure measurement in molecular epidemiology. Cancer Epidemiol Biomarkers Prev. 2005;14:184750 .

[2] Marchiori LL de M, Doi MY, Marchiori G de M, de Souza GV, Poli-Frederico RC, Ciquinato DS de A. Interleukin-1 alpha gene polymorphism (IL-1 $\alpha$ ) and susceptibility to tinnitus in the elderly. Noise Health. 2019;21:77-82.

[3] Jang YW, Gil KC, Lee JS, Kang WK, Park SY, Hwang KW. T-Cell Differentiation to T Helper 9 Phenotype is Elevated by Extremely Low-Frequency Electromagnetic Fields Via Induction of IL-2 Signaling. Bioelectromagnetics. 2019;40:588-601. 